OPEN ACCESS

Edited by:

Fabrizio Michetti,

Catholic University of the Sacred

Heart, Italy

Reviewed by:

José Antonio Pariente,

Universidad de Extremadura, Spain

Jiaxiang Chen,

Nanchang University, China

*Correspondence:

Fuwu Wang

wangfuwu@sdu.edu.cn

†These authors have contributed equally to this work

Received: 04 September 2019

Accepted: 03 February 2020

Published: 06 March 2020

Citation:

Liu C, Zhou W, Li Z, Ren J, Li X, Li S, Liu Q, Song F, Hao A and Wang F (2020) Melatonin Protects Neural Stem Cells Against Tri-Ortho-Cresyl

Phosphate-Induced Autophagy.

Front. Mol. Neurosci. 13:25. doi: 10.3389/fnmol.2020.00025

\section{Melatonin Protects Neural Stem Cells Against Tri-Ortho-Cresyl Phosphate-Induced Autophagy}

\author{
Chang Liu ${ }^{1 \dagger}$, Wenjuan Zhou ${ }^{1 \dagger}$, Zhaopei $\mathrm{Li}^{2}$, Jun Ren ${ }^{1}$, Xian $\mathrm{Li}^{1}$, Shan $\mathrm{Li}^{1}$, Qian Liu ${ }^{1}$, \\ Fuyong Song ${ }^{3}$, Aijun Hao ${ }^{1}$ and Fuwu Wang ${ }^{1 *}$ \\ ${ }^{1}$ Key Laboratory for Experimental Teratology of Ministry of Education, Shandong Key Laboratory of Mental Disorders, \\ Department of Anatomy and Histoembryology, School of Basic Medical Sciences, Shandong University, Jinan, China, \\ ${ }^{2}$ Department of Oncology, Shandong Provincial Hospital Affiliated to Shandong University, Jinan, China, ${ }^{3}$ Institute of \\ Toxicology, School of Public Health, Shandong University, Jinan, China
}

Tri-ortho-cresyl phosphate (TOCP) is an extensively used organophosphate in industry. It has been proven to lead to toxicity in different organ systems, especially in the nervous system. Neural stem cells (NSCs) play important roles in both embryonic and adult nervous systems. However, whether TOCP induces cytotoxicity in embryonic NSCs remains unclear. In this study, mouse NSCs were exposed to different concentrations of TOCP for $24 \mathrm{~h}$. The results showed that TOCP led to impaired proliferation of NSCs and induced the autophagy of NSCs by increasing the generation of intracellular reactive oxygen species (ROS) and decreasing the phosphorylation of extracellular regulated protein kinase (ERK1/2). Melatonin has been reported to exert neuroprotective effects via various mechanisms. Therefore, we further investigate whether melatonin has potential protective effects against TOCP-induced cytotoxicity on NSCs. Our data showed that melatonin pretreatment attenuated TOCP-induced autophagy by suppressing oxidative stress and restoring ERK1/2 phosphorylation consistently. Taken together, the results indicated that TOCP induced the autophagy in mouse NSCs, and melatonin may effectively protect NSCs against TOCP-induced autophagy.

\section{Keywords: tri-ortho-cresyl phosphate, neural stem cells, autophagy, melatonin, cytotoxicity}

\section{HIGHLIGHTS}

- Tri-ortho-cresyl phosphate (TOCP) suppressed neural stem cell (NSC) proliferation but not neural differentiation and cell apoptosis.

- Tri-ortho-cresyl phosphate induced the autophagy of NSCs by increasing reactive oxygen species (ROS) level and decreasing phosphorylated extracellular regulated protein kinase.

- Melatonin could effectively protect NSCs against TOCP-induced autophagy.

\section{INTRODUCTION}

Tricresyl phosphate (TCP), an extensively used organophosphate compound, widely acts as plasticizers, flame retardants, components of jet engine oil, and phosphorus-containing pesticides in industry, even in chemical fibers, textiles, and leather. TOCP is the most toxic compound among three isomers of TCP and has caused several poisoning incidents 
(Wolkoff et al., 2016; Lorke et al., 2017). TOCP has already been demonstrated to induce neurotoxicity, immunotoxicity (Brinkerhoff et al., 1981), and reproductive toxicity such as inhibition of spermatogonial stem cells and ovarian failure (Xu et al., 2016) in animals. Embryonic neurodevelopment plays an important role in the development of adult brain function. Among that, neural stem cells (NSCs) have the capability of self-renewal and differentiation for brain development. NSCs give rise to new neurons and glia throughout life and play key roles in both embryonic and adult nervous system (Gage and Temple, 2013). A number of studies proved that TOCP could damage structures and related functions of neurons (Chen et al., 2013; Song et al., 2015). Especially, TOCP was reported to cause severe and irreversible delayed neuropathy, namely, organophosphate-induced delayed neurotoxicity (OPIDN) in humans and sensitive animals. However, the effect of TOCP in NSCs in the developing brain remains unclear. Therefore, we investigated whether TOCP induced cytotoxicity on NSCs as well as its mechanisms.

Autophagy is an essential protein degeneration pathway to maintain intracellular homeostasis in eukaryotic cells. Under starving or stress conditions, autophagy is initiated to eliminate long-lived protein and damaged organelles by isolating these cytoplasmic components in autophagosomes and delivering them to lysosomes for degradation (Yorimitsu and Klionsky, 2005; He and Klionsky, 2009). Increasing evidence has shown that autophagy is involved in Alzheimer disease, Parkinson disease, Huntington disease, and other neurodegenerative diseases (Frake et al., 2015). Although autophagy was considered as a protective process when cells were faced with nutrition hunger or stress stimulation, some studies suggested that autophagy could also cause cell death, which is known as autophagic cell death (Levine and Kroemer, 2008). Recent studies showed that the neurotoxicity of TOCP on human neuroblastoma SH-SY5Y cells, as well as its reproductive toxicity on rat spermatogonial stem cells, was related to autophagy (Liu et al., 2015; Xu et al., 2017). In addition, TOCP was also reported to induce the autophagy of mouse Leydig TM3 cells in vitro via activating the oxidative stress (Liu et al., 2016). However, whether TOCP induces autophagy in NSCs and its potential mechanisms are unclear.

Melatonin is an endogenous hormone mainly secreted from the pineal gland in mammal brain (Reiter, 1991), which plays a critical role in physical activities including regulation of circadian rhythms and reproductive and neuroendocrine actions (Dubocovich, 2007; Hardeland, 2008). Recently, several studies have shown that melatonin could significantly decrease the production of ROS under various conditions acting as an endogenous free radical scavenger and antioxidant (Wang et al., 2013; Brazão et al., 2015; Torres et al., 2015). In addition, melatonin could also protect various cells through modulating multiple signaling pathways (Janjetovic et al., 2014; Yu et al., 2014; Lamont et al., 2015). For example, melatonin has been demonstrated to protect NSCs under pathological conditions by inhibiting the production of ROS and regulating the expression of signaling pathway proteins (Fu et al., 2011; Song et al., 2015). Moreover, melatonin was reported to be involved in the cell protection by inhibiting the autophagy (Pi et al., 2015; Yoo et al., 2016). However, whether melatonin has a protective effect on TOCP-treated NSCs is still unknown.

Therefore, the purpose of the current study was to explore the effects of TOCP on NSCs, the protective role of melatonin on the TOCP-induced toxicity of NSCs, and the underlying molecular mechanisms. We report here that melatonin pretreatment significantly attenuated TOCP-induced autophagy of NSCs, at least in part, by suppressing oxidative stress and consistently restoring extracellular regulated protein kinase (ERK1/2) signaling pathway.

\section{MATERIALS AND METHODS}

\section{Materials}

TOCP (purity >99\%) was obtained from BDH Chemicals Company Limited (Poole, UK). Dulbecco modified Eagle medium (DMEM)/F12 (1:1) medium and B27 supplement were purchased from Gibco BRL (Caithersburg, MD, USA). Basic fibroblast growth factor (bFGF) was purchased from R\&D Systems, Minneapolis, MN, USA. Bafilomycin $\mathrm{A}_{1}$ (Baf $\mathrm{A}_{1}$ ), N-acetylcysteine (NAC), melatonin, and 4,5-dimethyl2-thiazolyl)-2,5-diphenyl-2-H-tetrazolium bromide (MTT) were purchased from Sigma-Aldrich (St. Louis, MO, USA). Cell-Light ${ }^{\mathrm{TM}}$ 5-ethynyl-2'-deoxyuridine (EdU) Apollo ${ }^{\circledR} 488$ in vitro Imaging Kit (100T) was purchased from RiboBio Company Limited (Guangzhou, China). Annexin V-fluorescein isothiocyanate (FITC)/propidium iodide (PI) Apoptosis Detection Kit was purchased from Abcam, Cambridge, MA, USA. The primary antibodies: rabbit anti-light chain 3 beta (LC3B), rabbit anti-neuronal class III $\beta$-tubulin (Tuj-1), and rabbit anti-glial fibrillary acidic protein (GFAP) were purchased from Cell Signaling Technology, Danvers, MA, USA. Monoclonal anti- $\beta$-actin, goat anti-rabbit immunoglobulin $G$ (IgG), and anti-mouse IgG were purchased from Sigma-Aldrich (St. Louis, MO, USA). Bicinchoninic acid assay protein assay kit was purchased from Pierce Biotechnology Inc., Rockford, IL, USA. The $2^{\prime}, 7^{\prime}$-dichlorodihydro-fluorescein diacetate $\left(\mathrm{H}_{2}\right.$ DCFDA $)$ and dihydroethidium (DHE) were purchased from Molecular Probes, Eugene, OR, USA.

\section{Cell Culture}

The primary NSCs were isolated and cultured according to a previously described method with minor modifications ( $\mathrm{Fu}$ et al., 2011; Chen et al., 2016). NSCs were initially derived from embryonic brain of Kunming mice at embryonic day 12.5. The entire cerebrum was separated from embryonic brain and then was placed into ice-cold Hanks balanced salt solution. Following mechanical separation, cells were centrifuged, resuspended, and incubated with DMEM/F12(1:1) medium plus $2 \% \mathrm{~B} 27,20 \mathrm{ng} / \mathrm{ml}$ bFGF, $100 \mathrm{U} / \mathrm{ml}$ penicillin, and $100 \mathrm{mg} / \mathrm{ml}$ streptomycin at $37^{\circ} \mathrm{C}$ in a humidified atmosphere of $5 \% \mathrm{CO}_{2}$. The culture medium was replaced, and NSCs were mechanically separated again every 2 days. Animal care and treatment complied with the National Institutes of Health Guide for the Care and Use of Laboratory Animals, and the animal experiments were approved by the 
Institutional Animal Care and Use Committees of Shandong University (No. 201402020).

\section{Cell Treatment}

NSCs at two to four passages were collected by centrifuging at $600 \mathrm{~g}$ for $5 \mathrm{~min}$ and resuspended in medium with $0-100 \mu \mathrm{M}$ TOCP. Meanwhile, $10 \mathrm{nM} \mathrm{Baf}_{1}, 5 \mu \mathrm{M}$ NAC, $50 \mu \mathrm{M}$ PD98059 (specific inhibitor of ERK), or $40 \mu \mathrm{M}$ melatonin was added into the medium before TOCP treatment, respectively. The cells were then cultivated for another $24 \mathrm{~h}$ and were processed for further research.

\section{MTT Assay}

The cell viability of NSCs was detected by MTT assay. The NSCs were seeded into 96-well plates (Corning Inc., Corning, NY, USA) preincubated with poly-L-lysine (PLL). The density of cells was $5 \times 10^{4}$ cells/well with $200 \mu \mathrm{l}$ culture medium per well. After the cells were adherent, the NSCs were treated with 0-100 $\mu \mathrm{M}$ TOCP for $24 \mathrm{~h}$ in the presence or absence of $10 \mathrm{nM}$ Baf $\mathrm{A}_{1}, 5$ $\mu \mathrm{M}$ NAC, and $40 \mu \mathrm{M}$ melatonin, respectively. Then, $20 \mu \mathrm{MTT}$ $(0.5 \mathrm{mg} / \mathrm{ml})$ was added into each well for another $4 \mathrm{~h}$, and the culture medium was replaced by $150 \mu \mathrm{l}$ DMSO per well. The absorbance was determined by spectrophotometer at $490 \mathrm{~nm}$.

\section{EdU Assay}

Cell proliferation activity was determined by EdU assay according to the manufacturer's instructions. Briefly, the NSCs were planted on glass cover slips pretreated with PLL and were exposed to $0-100 \mu \mathrm{M}$ TOCP for $24 \mathrm{~h}$. Cells were incubated with EdU reagent at room temperature for $1 \mathrm{~h}$ and washed with phosphate-buffered saline three times. The photographs were taken by a fluorescence microscope (IX71; Olympus, Tokyo, Japan).

\section{Western Blot Analysis}

The differently treated NSCs were collected, washed with cold phosphate-buffered saline, and lysed with cold RIPA buffer. After incubation at $4^{\circ} \mathrm{C}$ for $30 \mathrm{~min}$, the samples were centrifuged at $11,000 \mathrm{~g}$ for $10 \mathrm{~min}$ at $4^{\circ} \mathrm{C}$. The protein concentration was detected by a BCA protein assay kit (Pierce Biotechnology Inc., Rockford, IL, USA). Samples were loaded on to sodium dodecyl sulfate-polyacrylamide gel electrophoresis, separated on $8 \%$ to $15 \%$ gradient gels, and electrophoretically transferred to polyvinylidene fluoride membranes. After transfer, the membranes were blocked with $5 \%$ fat-free milk for $90 \mathrm{~min}$ at room temperature. The membranes were then incubated with specific primary antibodies $(1: 1,000)$, at $4^{\circ} \mathrm{C}$ overnight, respectively. After three washes with tris-buffered saline tween-20 (TBST) buffer, the membranes were incubated with the corresponding secondary antibody $(1: 10,000)$ at room temperature for $1 \mathrm{~h}$, respectively. Monoclonal anti- $\beta$-actin acts as an internal control. The membranes were finally incubated with ECL reagents, and immunoreactive bands were detected using the Image-Pro Plus 6.0 software (Media Cybernetics, Inc., Rockville, MD, USA).

\section{Annexin V-FITC/PI Staining Assay}

The NSCs with different treatments were collected in a single-cell suspension in $500 \mu \mathrm{l}$ binding buffer per sample at $37^{\circ} \mathrm{C}$, and $5 \mu \mathrm{l}$ annexin V-FITC and $5 \mu \mathrm{l}$ PI were added into the medium, respectively, according to the manufacturer's instructions. The results were analyzed by flow cytometry within $1 \mathrm{~h}$.

\section{Transmission Electron Microscopy Analysis}

After treatment with $0-100 \mu \mathrm{M}$ TOCP for $24 \mathrm{~h}$, NSCs were harvested and centrifuged. The supernatant was replaced by cold $2.5 \%$ glutaraldehyde for $2 \mathrm{~h}$ and osmium tetroxide (OsO4) for $1 \mathrm{~h}$. After the protocols of dehydration and embedding, double stain was administered on ultrathin sections $(60 \mathrm{nM})$ with uranyl acetate and lead citrate, and the observation was taken by transmission electron microscope (TEM).

\section{ROS Assay}

NSCs $\left(1 \times 10^{6}\right.$ cells/well $)$ were cultured in six-well culture plates pretreated with PLL. After various treatments, the cells were incubated with $10 \mu \mathrm{M} \mathrm{H} \mathrm{H}_{2}$ DCFDA and $2 \mu \mathrm{M}$ DHE for $30 \mathrm{~min}$ at $37^{\circ} \mathrm{C}$, respectively. Following counterstaining by $4^{\prime}$, 6-diamidino-2-phenylindole (DAPI), the images were taken with fluorescence microscope (IX71; Olympus). Meanwhile, the fluorescence intensity of the samples was determined by flow cytometry, respectively.

\section{Statistical Analysis}

The data are expressed as mean \pm SD of at least three independent experiments. The values were analyzed by one-way analysis of variance with SPSS 13.0 software (International Business Machines Corporation, Armonk, NY, USA). $P<0.05$ was considered statistically significant.

\section{RESULTS}

\section{Effect of TOCP on the Cell Viability of Mouse NSCs}

First, the effect of different concentrations of TOCP $(0-100 \mu \mathrm{M})$ on NSC cell viability was investigated by MTT assay. As shown in Figure 1, TOCP decreased the cell viability of NSCs in a dose-dependent manner. According to the MTT results, two concentrations of TOCP (40 and $100 \mu \mathrm{M}$ ) were used in the following study to detect the effect on NSCs.

\section{Effect of TOCP on Proliferation, Differentiation, and Cell Apoptosis of Mouse NSCs}

To further assess the characteristics of NSCs under TOCP exposure condition, a series of experiments were conducted. First, the EdU assay was performed to detect the effect of TOCP (40 and $100 \mu \mathrm{M}$ for $24 \mathrm{~h}$ ) on the proliferation of NSCs, and the results showed that TOCP treatment significantly decreased the percentage of EdU-positive cells compared with the control (Figure 2A, $P<0.05$ and $P<0.01,40$ and $100 \mu \mathrm{M}$ TOCP, respectively). The results suggest that the proliferation 


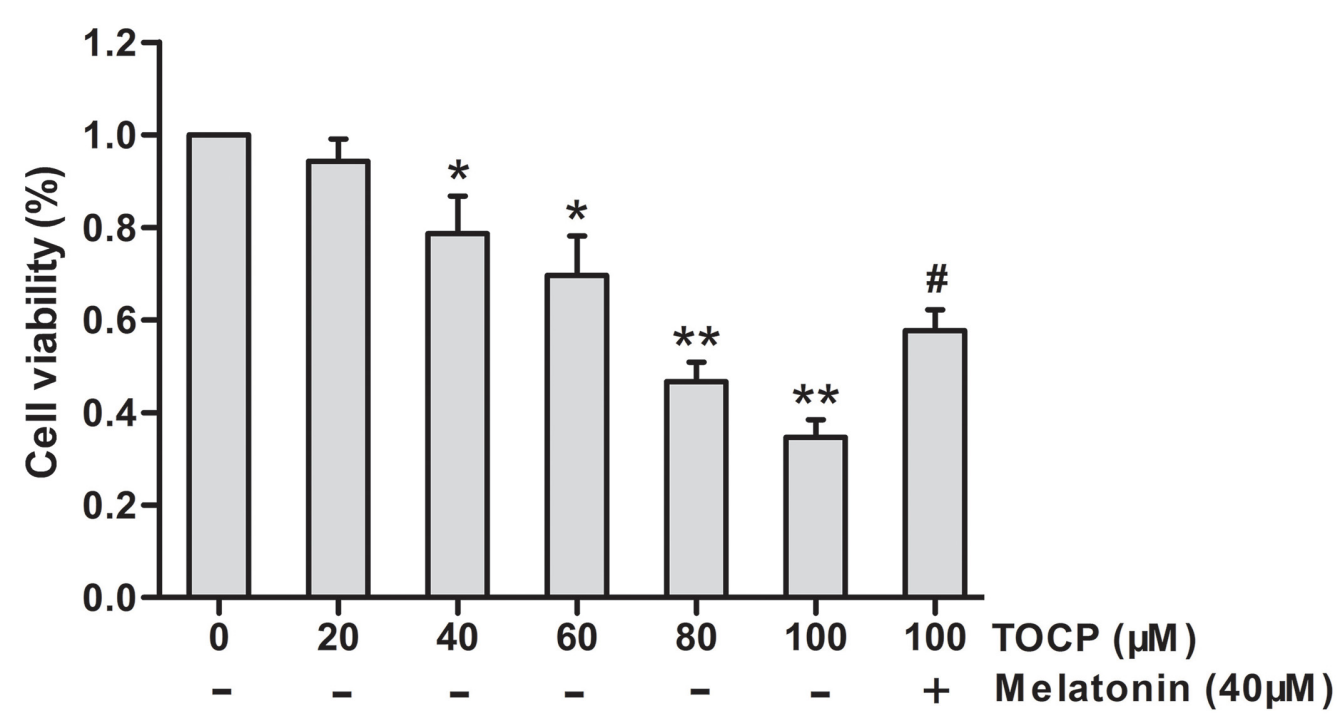

FIGURE 1 | Effects of tri-ortho-cresyl phosphate (TOCP) on the cell viability of mouse neural stem cells (NSCs). NSCs were treated with different concentrations of TOCP $(0-100 \mu \mathrm{M})$ with or without pretreatment by $40 \mu \mathrm{M}$ melatonin for 30 min. The cell viability was detected by $\mathrm{MTT}$ assay. Data are expressed as mean $\pm \mathrm{SD}$ of at least three independent experiments. ${ }^{\star} P<0.05$, ${ }^{\star \star} P<0.01$ vs. control; ${ }^{*} P<0.05$ vs. $100 \mu \mathrm{M}$ TOCP alone.

of NSCs was suppressed under TOCP exposure condition in the present study.

Then, the effect of TOCP on the neural differentiation of NSCs was observed by detecting the expression of differentiation-associated proteins. Western blot revealed that TOCP did not significantly change the protein expression levels of Tuj-1 (a neuronal marker) and GFAP (an astrocyte marker) in NSCs (Figure 2B), which implied that TOCP had no significant effects on the neural differentiation of NSCs.

Finally, the cell apoptosis of NSCs induced by TOCP was examined using annexin V-FITC/PI double-staining method. Annexin V and PI can be used to distinguish early and late apoptosis cells and dead cells. The flow cytometry results displayed that both 40 and $100 \mu \mathrm{M}$ TOCP did not significantly change the apoptotic or necrotic ratio of NSCs compared with the control, and there was also no significant difference of apoptotic ratio between 40 and $100 \mu \mathrm{M}$ TOCP groups (Figure 2C). In addition, similar results were obtained by detecting the expression of apoptosis-associated protein, cleaved caspase-3 (Figure 2D). Our data suggest that TOCP also did not significantly affect the cell apoptosis of NSCs in the concentration ranges of the present study.

\section{Melatonin Attenuates TOCP-Induced Autophagy in Mouse NSCs}

To explore whether autophagy is involved in the toxicity of TOCP on NSCs, the formation of autophagosomes was first detected by TEM. The results showed that the number of autophagosomes significantly increased in NSCs treated with 100 $\mu \mathrm{M}$ TOCP for $24 \mathrm{~h}$ (Figure 3A). Meanwhile, autophagy-related protein LC3B was also analyzed by Western blot. As shown in Figure 3B, $100 \mu \mathrm{M}$ TOCP markedly increased the ratio of LC3-II to LC3-I in NSCs, which was consistent with the results of TEM.
To further determine whether the increase of autophagosomes was due to the enhancement of autophagy activity or the block of autophagy flux in TOCP-treated NSCs, $10 \mathrm{nM} \mathrm{Baf} \mathrm{A}_{1}$ (a specific inhibitor of autophagy at a latest stage) was preadministered for $30 \mathrm{~min}$ into the medium before $100 \mu \mathrm{M}$ TOCP exposure for $24 \mathrm{~h}$. The results showed that Baf $A_{1}$ pretreatment further increased both the number of autophagosomes and the ratio of LC3-II to LC3-I compared to TOCP treatment alone (Figures $\mathbf{3 A}, \mathbf{B}$ ), which implied that TOCP indeed enhanced the complete autophagy flux in NSCs.

To investigate the effect of melatonin on TOCP-treated NSCs, we first analyzed the cell viability of NSCs by MTT assay. The results showed that melatonin $(40 \mu \mathrm{M})$ pretreatment for $30 \mathrm{~min}$ before TOCP exposure significantly restored the cell viability of NSCs compared with $100 \mu \mathrm{M}$ TOCP treatment alone (Figure 1, $P<0.05$ ). Then, the protective effects of melatonin on TOCP-induced autophagy were detected. The TEM results displayed that melatonin pretreatment could significantly reduce the number of autophagosomes (Figure 3A). Western blot also showed that melatonin markedly decreased the ratio of LC3-II to LC3-I (Figure 3B). The results implied that melatonin could inhibit the autophagy induced by TOCP in NSCs.

\section{Melatonin Decreases the Production of ROS Induced by TOCP in Mouse NSCs}

Previous studies have shown that TOCP could trigger the oxidative stress and autophagy (Liu et al., 2016). To determine whether oxidative stress is relevant to TOCP-treated NSCs, the intracellular ROS level was examined by $\mathrm{H}_{2}$ DCFDA and DHE assay, respectively (Figure 4A). Fluorescence photomicrography and flow cytometry analysis showed that TOCP significantly increased the intracellular ROS production in NSCs (Figures 4A,B). Then, $N$-acetyl-L-cysteine (NAC, 


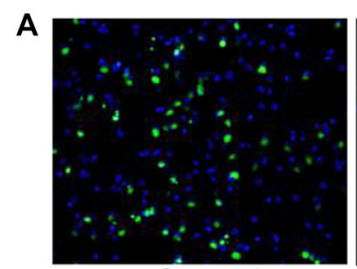

Control

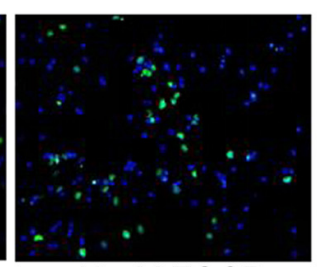

$40 \mu \mathrm{M}$ TOCP

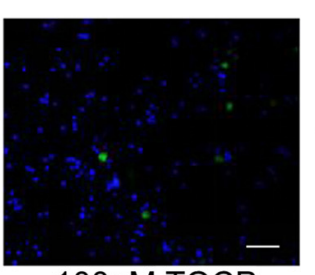

$100 \mu \mathrm{M}$ TOCP

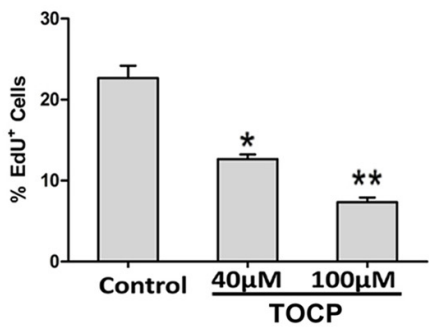

GFAP
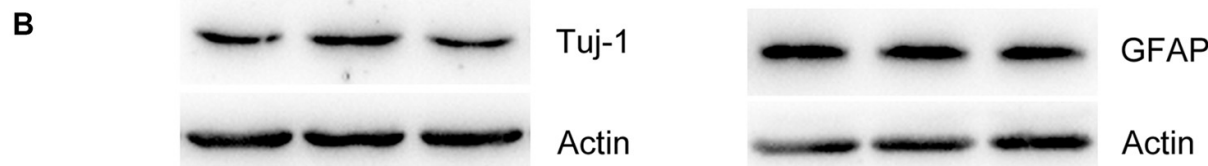

Control $40 \mu \mathrm{M} 100 \mu \mathrm{M}$

Control $\frac{40 \mu \mathrm{M} 100 \mu \mathrm{M}}{\mathrm{TOCP}}$
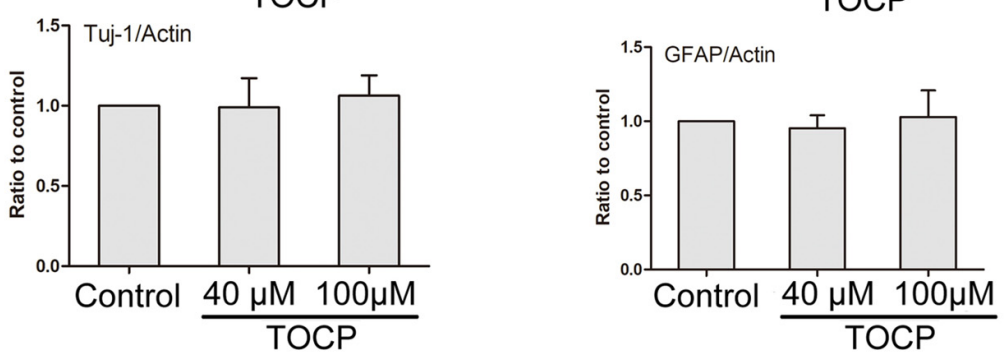

C
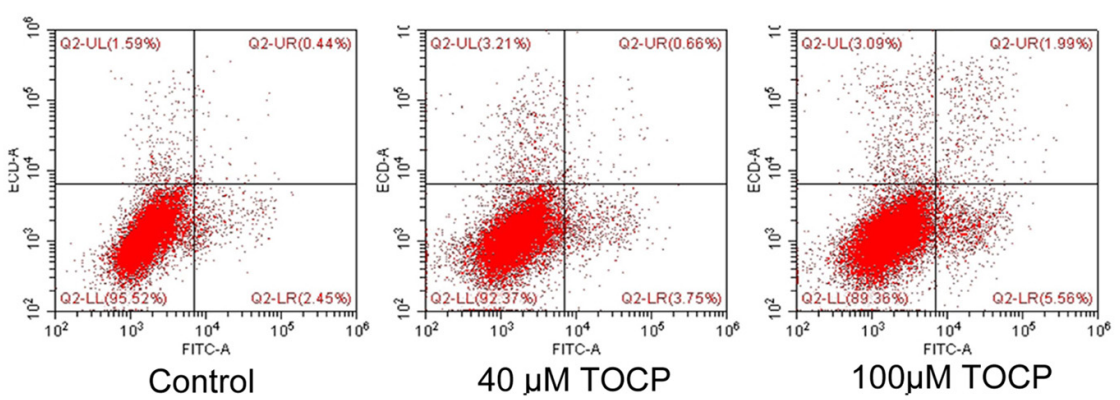

D

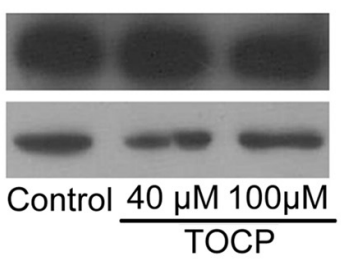
cleaved caspase-3

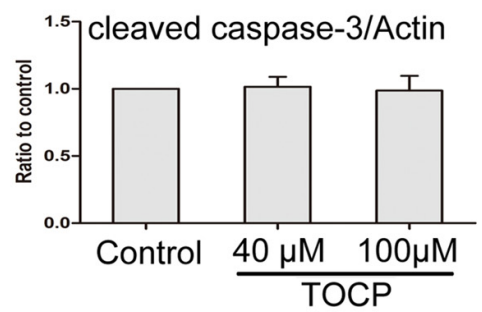

FIGURE 2 | Effects of TOCP on proliferation, differentiation, and cell apoptosis of mouse NSCs. NSCs were treated with TOCP (40 and $100 \mu$ M, respectively). (A) The cell proliferation was determined by EdU assay. Scale bar, $50 \mu \mathrm{m}$. (B) The neural differentiation-related proteins Tuj-1 (a neuronal marker) and glial fibrillary acidic protein (GFAP; an astrocyte marker) were detected by Western blot. (C) The cell apoptosis of TOCP-treated NSCs was assessed using annexin V-FITC/PI double staining by flow cytometry. (D) The apoptosis-related protein, cleaved caspase-3 was examined by Western blot. Data are presented as mean \pm SD of at least three independent experiments. ${ }^{\star} P<0.05$, ${ }^{\star \star} P<0.01$ vs. control.

an inhibitor of oxidative stress, $5 \mu \mathrm{M}$ ) was added into the medium for $30 \mathrm{~min}$ before TOCP exposure. The results showed that NAC pretreatment markedly suppressed the ROS level in NSCs (Figures 4A,B) and reduced the ratio of LC3-II to LC3-I in NSCs (Figure 4C), which suggested that TOCP induced autophagy by elevating the oxidative level in NSCs. Finally, melatonin was added into the medium for $30 \mathrm{~min}$ before TOCP exposure, and the results showed that melatonin significantly inhibited the intracellular ROS level in NSCs (Figures 4A,B). These results suggested that melatonin could inhibit the TOCP-induced autophagy in NSCs through suppressing the oxidative stress. 

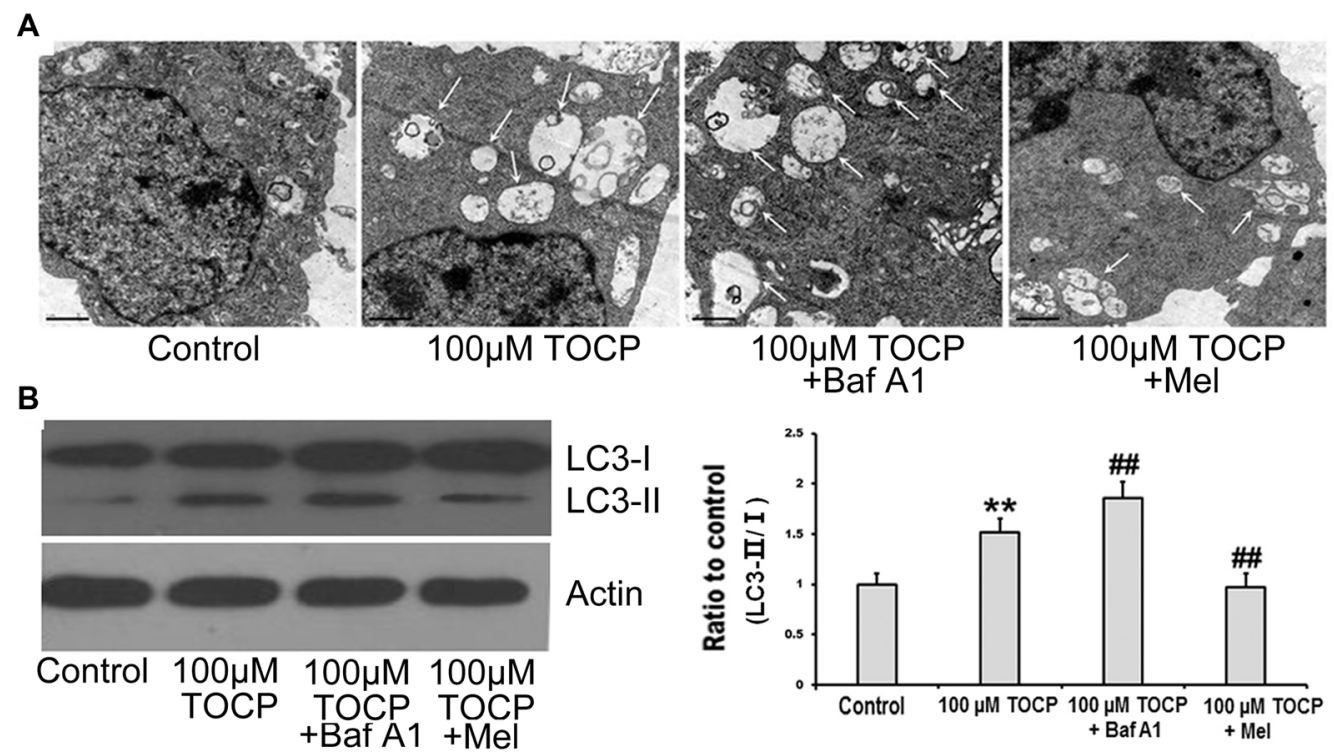

FIGURE 3 | Melatonin attenuates TOCP-induced autophagy in mouse NSCs. NSCs were treated with $100 \mu \mathrm{M}$ TOCP with or without the pretreatment of 10 nM bafilomycin $\mathrm{A}_{1}$ (Baf $\mathrm{A}_{1}$ ) and $40 \mu \mathrm{M}$ melatonin, respectively. (A) The formation of autophagosomes was detected by transmission electron microscope (TEM). Scale bar, $1 \mu \mathrm{m}$. (B) Autophagy-related protein LC3B was analyzed by Western blot. Data are expressed as mean \pm SD of at least three independent experiments. ${ }^{* \star} P<0.01$ vs. control, ${ }^{\# \#} P<0.01$ vs. $100 \mu \mathrm{M}$ TOCP.

\section{Melatonin Activates ERK1/2 Signaling Pathway Suppressed by TOCP in Mouse NSCs}

Several studies proved that ERK1/2 signaling protein participates in the autophagy of several types of cells (Jo et al., 2014; Huang et al., 2015). To further determine whether ERK1/2 signaling protein is involved in TOCP-induced autophagy of NSCs, the phosphorylation of ERK1/2 (p-ERK) was examined in NSCs treated with $100 \mu \mathrm{M}$ TOCP for $24 \mathrm{~h}$. As shown in Figure $\mathbf{5 A}$, TOCP significantly decreased the expression of p-ERK $(P<0.01)$. Pretreatment with $50 \mu \mathrm{M}$ PD98059 (specific ERK1/2 inhibitor) for 30 min significantly reduced the p-ERK level and increased the ratio of LC3-II to LC3-I (Figures 5A,B). The results suggested that TOCP may also induce the autophagy of NSCs by inhibiting the phosphorylation of ERK1/2 protein. Melatonin pretreatment significantly increased the p-ERK1/2 level compared with the TOCP treatment alone (Figure 5A). Furthermore, melatonin treatment also significantly decreased the ratio of LC3-II to LC3-I in NSCs (Figure 3B). These results suggested that melatonin could suppress the autophagy in NSCs treated with TOCP, at least partially, by restoring the phosphorylation of ERK1/2 protein.

\section{DISCUSSION}

Increasing studies have shown that TOCP induces the autophagy in many types of cells (Long et al., 2014; Song et al., 2014; Liu et al., 2016). However, it is still unclear whether TOCP has an effect on NSCs. The major finding of the present study was that TOCP induced the autophagy in NSCs and decreased the cell proliferation of NSCs, whereas melatonin protected the NSCs against TOCP-induced autophagy through suppressing the oxidative stress and activating the ERK1/2 signaling pathway.

TOCP has been extensively used in industry serving as pesticides, plasticizers, lubricants, additives, and chemical warfare agents. In addition to poisoning of acute organophosphorus compounds, TOCP can also cause severe and irreversible delayed neuropathy, OPIDN, in sensitive animals and humans. Although the OPIDN incidents were first reported in the 1930's in America, there are still various theories on the pathogenesis of OPIDN (Wolkoff et al., 2016; Lorke et al., 2017). Current clinical treatments have no specific effects, and the prognosis has been poor until now, which not only significantly influenced patient health but also caused a heavy societal burden (Abdollahi and Karami-Mohajeri, 2012; Emerick et al., 2012).

NSCs exist in both the embryonic and adult nervous system and will differentiate into neurons and glia. NSC therapy has been regarded as a potential therapy for neurological diseases such as neurodegeneration, stroke, or spinal cord lesions (Gage and Temple, 2013; Tsukamoto et al., 2013). Recent studies have shown that TOCP inhibited the formation of axon-like processes in N2a and PC12 cells (Flaskos et al., 1998) and decreased the cell viability in series of cells such as neuroblastoma SH-SY5Y cells and mouse spermatogonial stem cells (Long and $\mathrm{Wu}, 2008$; Liu et al., 2016). Previous studies have focused on the neurotoxicity of TOCP to neurons, but no studies have considered whether TOCP has effects on NSCs, as well as its potential mechanisms. Here, the present study demonstrated that TOCP suppressed cell viability and inhibited the proliferation of NSCs, but it had no significant effects on the differentiation and apoptosis of NSCs. 

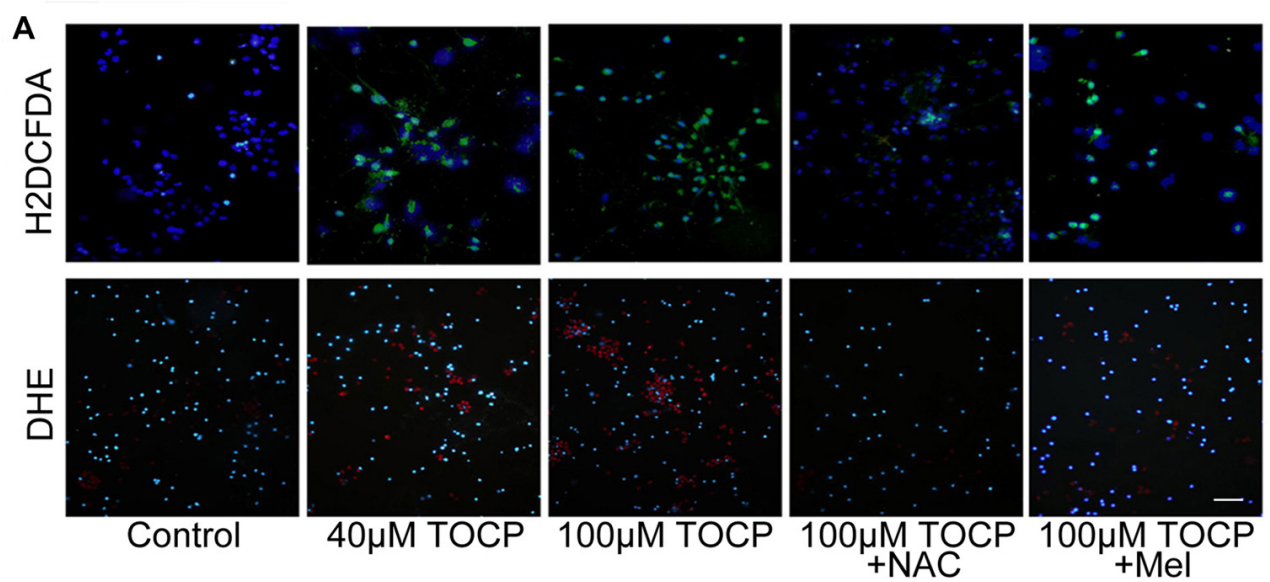

B
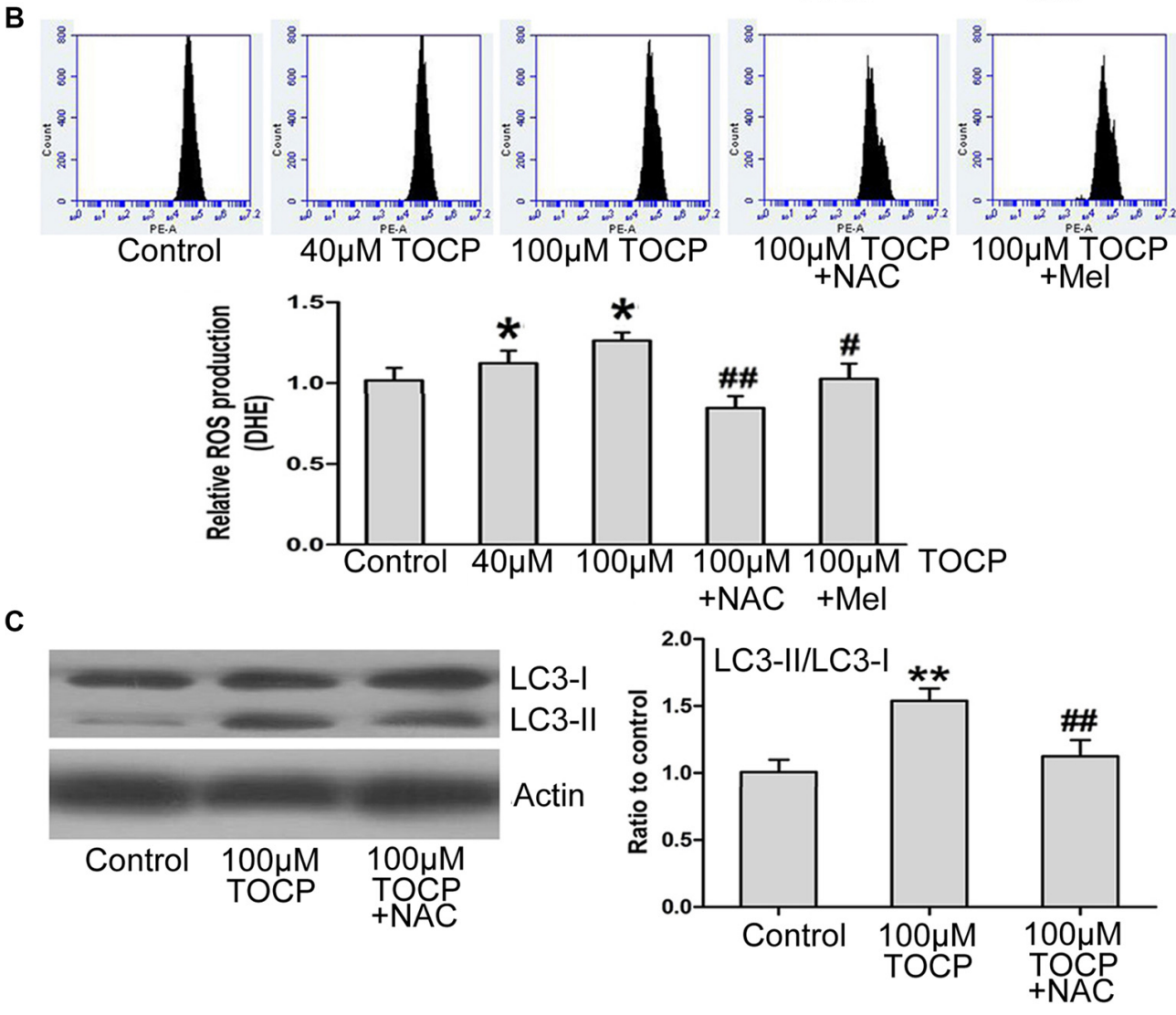

C

FIGURE 4 | Melatonin decreases the production of reactive oxygen species (ROS) induced by TOCP in mouse NSCs. NSCs were exposed to different concentrations of TOCP (40 or $100 \mu \mathrm{M}$ ) with or without $5 \mu \mathrm{M}$ NAC and $40 \mu \mathrm{M}$ melatonin, respectively, before TOCP exposure. (A) The intracelllular ROS level was examined by H2DCFDA (green) and DHE (red) assay. The cells were then counterstained by DAPI (blue). Scale bar, $50 \mu \mathrm{m}$. (B) The results of DHE staining were also observed by flow cytometry. (C) NSCs were pretreated with $5 \mu \mathrm{M}$ NAC before $100 \mu \mathrm{M}$ TOCP exposure, and autophagy-related protein, LC3, was analyzed by Western blot. Data are expressed as mean $\pm \mathrm{SD}$ of three individual experiments. ${ }^{\star} P<0.05,{ }^{* \star} P<0.01$ vs. control, ${ }^{\#} P<0.05$, ${ }^{\# \#} P<0.01 \mathrm{vs}$. $100 \mu \mathrm{M}$ TOCP alone.

The results were similar with the previous report that TOCP markedly inhibited the viability of mouse Leydig TM3 cells and had no effects on its apoptosis (Liu et al., 2016).

Autophagy is a highly conserved intracellular catabolic progress, which eliminates damaged organelles and long-lived proteins by isolating these cytoplasmic components in autophagosomes and delivering them to lysosomes for degradation. In recent years, many standard methods were used to monitor the autophagy including detecting the formation of autophagosomes and the change of LC3-II/I ratio (Klionsky et al., 2008). Transmission electron microscopy was widely used to observe the autophagosome formation and analyze 

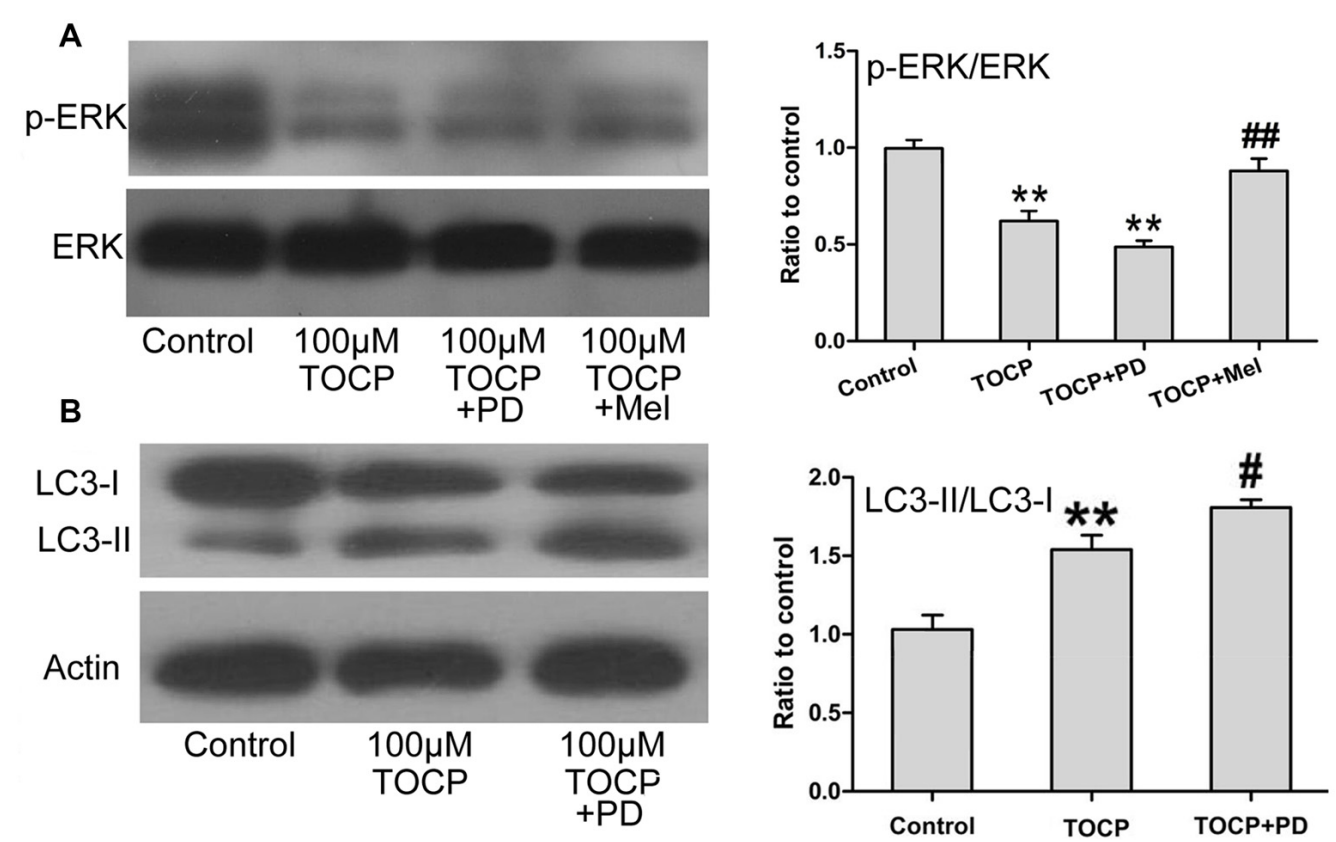

FIGURE 5 | Melatonin activates ERK1/2 signaling pathway suppressed by TOCP in mouse NSCs. NSCs were treated with $100 \mu \mathrm{M}$ TOCP in the presence or absence of $50 \mu \mathrm{M}$ PD98059 (a specific ERK1/2 inhibitor) and $40 \mu \mathrm{M}$ melatonin, respectively. (A) The expression of p-ERK in TOCP-treated NSCs was examined by Western blot. (B) Autophagy-related protein, LC3, was analyzed by Western blot. Data are expressed as mean \pm SD of at least three independent experiments. ${ }^{\star \star} P<0.01$ vs. control, ${ }^{\#} P<0.05,{ }^{\# \#} P<0.01$ vs. $100 \mu \mathrm{M}$ TOCP alone.

both the qualitative and quantitative change of autophagy. In the present study, the results of TEM showed that TOCP treatment remarkably increased the number of autophagosomes in NSCs. In addition, LC3 protein was also extensively used to detect the level of autophagy. LC3 is initially synthesized in an unprocessed form, proLC3, which then is transformed to a proteolytically processed form, LC3-I, and is finally modified into the phosphatidylethanolamine-conjugated form, LC3-II (Sou et al., 2006). LC3-II is considered as the marker associated with completed autophagosomes, and the ratio of LC3-II to LC3-I becomes one of the gold standards for detecting the autophagy level. The present study also demonstrated that TOCP exposure significantly enhanced the ratio of LC3-II to LC3-I in NSCs. Both the TEM and LC3 detecting results suggest that TOCP indeed induced the cell autophagy of NSCs.

It is well known that autophagy is actually a process including the formation of autophagosomes, the fusion between autophagosomes and lysosomes, and the formation of autolysosomes, which is also called autophagic flux. It was reported that the accumulation of autophagosomes may be due to the increased autophagic activity or the reduced turnover of autophagosomes (Klionsky et al., 2008). Therefore, to further explore the effects of TOCP on the autophagy of NSCs, Baf $A_{1}$, a specific inhibitor of autophagy at the latest stage by inhibiting the fusion between autophagosomes and lysosomes, was used in the present study. Bafilomycin $A_{1}$ pretreatment further obviously enhanced the ratio of LC3-II to LC3-I in NSCs treated with TOCP. This demonstrated that TOCP treatment increased the overall autophagic flux in NSCs, and Baf $\mathrm{A}_{1}$ blocked the autophagic flux, which resulted in the accumulation of LC3-II and higher ratio of LC3-II to LC3-I. The findings further proved that TOCP could induce the autophagy and enhance the autophagic flux in NSCs.

Despite the considerable advances in the biology of autophagy, the functions of autophagy are still unclear. Previous studies showed that oxidative stress could induce autophagy in both humans and animals (Filomeni et al., 2015). Both oxidative stress and autophagy pathways are all relevant mechanisms of toxicity from organophosphorus compounds treatment (Kovacic, 2003; Xu et al., 2017). Moreover, some studies showed that oxidative stress was involved in TOCP-induced autophagy of mouse Leydig TM3 cells (Liu et al., 2016). However, it is unknown whether oxidative stress plays a critical role in TOCP-induced autophagy of NSCs. The present results showed that TOCP significantly activated the oxidative stress and induced the autophagy in NSCs. Meanwhile, the treatment of specific oxidative stress inhibitor NAC markedly decreased the level of oxidative stress and reduced the autophagy. Melatonin, one of the strongest antioxidants, has been reported to be involved in regulating the autophagy induced by oxidative stress, endoplasmic reticulum stress, and mitochondria dysfunction (Fernández et al., 2015; Wang et al., 2015). The current study showed that melatonin also significantly decreased TOCP-induced oxidative stress in NSCs and the ratio of LC3-II to LC3-I. These results demonstrated that TOCP could significantly increase the level of oxidative stress in NSCs, and the higher oxidative stress level in turn induced the autophagy 
of NSCs; meanwhile, melatonin could protect the TOCP-treated NSCs by inhibiting oxidative stress.

In addition to oxidative stress, multiple signaling pathways including ERK1/2 signaling proved to play important roles in cell autophagy (Parzych and Klionsky, 2014). In the present study, our results showed that TOCP decreased the expression of p-ERK in NSCs, and the specific ERK1/2 inhibitor PD98059 pretreatment further decreased the expression of $\mathrm{p}$-ERK and increased the ratio of LC3-II to LC3-I in TOCP-treated NSCs. Melatonin has strong antioxidant characteristics and is involved in the cell autophagy by modulating series of signaling pathways (Yoo et al., 2016; Zhang et al., 2016). The present study showed that melatonin significantly increased the expression of p-ERK in NSCs and decreased the autophagy of NSCs, which was consistent with previous results that melatonin could protect cells against pathological condition-induced autophagy through regulating the ERK1/2 signaling pathway (Yoo et al., 2016; Zhang et al., 2016).

\section{CONCLUSION}

Numerous investigations have been conducted on TOCP toxicity, which include various mechanism studies such as acetyl cholinesterase inhibition, neuropathy target esterase inhibition, or $\gamma$-aminobutyric acid antagonization (Lorke et al., 2017) in the neural system (Craig and Barth, 1999; Zhang et al., 2007), immune system (Foil et al., 1980; Brinkerhoff et al., 1981), or reproductive system (Xu et al., 2016), but consensus regarding this topic has not been reached. The definite effects and potential mechanisms of TOCP on NSCs, which are essential in the nervous system, still remain to be explored and verified. This study demonstrated that TOCP induced the autophagy of NSCs and decreased cell viability and proliferation. In addition, melatonin could significantly suppress TOCP-induced

\section{REFERENCES}

Abdollahi, M., and Karami-Mohajeri, S. (2012). A comprehensive review on experimental and clinical findings in intermediate syndrome caused by organophosphate poisoning. Toxicol. Appl. Pharmacol. 258, 309-314. doi: 10.1016/j.taap.2011.11.014

Brazão, V., Colato, R. P., Santello, F. H., Filipin Mdel, V., Toldo, M. P., do Vale, G. T., et al. (2015). Interleukin-17, oxidative stress, and inflammation: role of melatonin during Trypanosoma cruzi infection. J. Pineal Res. 59, 488-496. doi: $10.1111 /$ jpi.12280

Brinkerhoff, C. R., Sharma, R. P., and Bourcier, D. R. (1981). The effects of tri-otolyl phosphate (TOTP) on the immune system of mice. Ecotoxicol. Environ. Saf. 5, 368-376. doi: 10.1016/0147-6513(81)90010-5

Chen, X., Du, Z., Li, X., Wang, L., Wang, F., Shi, W., et al. (2016). Protein palmitoylation regulates neural stem cell differentiation by modulation of EID1 activity. Mol. Neurobiol. 53, 5722-5736. doi: 10.1007/s12035-0159481-y

Chen, J. X., Sun, Y. J., Wang, P., Long, D. X., Li, W., Li, L., et al. (2013). Induction of autophagy by TOCP in differentiated human neuroblastoma cells lead to degradation of cytoskeletal components and inhibition of neurite outgrowth. Toxicology 310, 92-97. doi: 10.1016/j.tox.2013.05.012

Craig, P. H., and Barth, M. L. (1999). Evaluation of the hazards of industrial exposure to tricresyl phosphate: a review and interpretation autophagy of NSCs by inhibiting the oxidative stress level and activating the ERK1/2 signaling pathway and consequently restore cell viability of NSCs. Our study may contribute to forming a more comprehensive and systematic understanding of the toxicity of TOCP, especially in the nervous system, and serving as a reference for clinical treatment of TOCP poisoning cases.

\section{DATA AVAILABILITY STATEMENT}

All datasets generated for this study are included in the article.

\section{ETHICS STATEMENT}

The animal study was reviewed and approved by Animal care and treatment complied with the National Institutes of Health Guide for the Care and Use of Laboratory Animals and the animal experiments were approved by the Institutional Animal Care and Use Committees of Shandong University (No. 201402020).

\section{AUTHOR CONTRIBUTIONS}

$\mathrm{CL}$ and WZ contributed to the conception and design of the study. ZL, JR, XL, SL and QL organized the database. FS performed the statistical analysis. CL, WZ, and $\mathrm{AH}$ wrote the first draft of the manuscript. FW wrote sections of the manuscript. All authors contributed to manuscript revision, read and approved the submitted version.

\section{FUNDING}

This study was funded by the National Natural Science Foundation of China (Grant Number 81271451); and the Key Research and Development Program of Shandong Province (Grant Number 2017GSF218094).

of the literature. J. Toxicol. Environ. Health B Crit. Rev. 2, 281-300. doi: 10.1080/109374099281142

Dubocovich, M. L. (2007). Melatonin receptors: role on sleep and circadian rhythm regulation. Sleep Med. 8, 34-42. doi: 10.1016/j.sleep.2007. 10.007

Emerick, G. L., DeOliveira, G. H., dos Santos, A. C., and Ehrich, M. (2012). Mechanisms for consideration for intervention in the development of organophosphorus-induced delayed neuropathy. Chem. Biol. Interact. 199, 177-184. doi: 10.1016/j.cbi.2012.07.002

Fernández, A., Ordóñez, R., Reiter, R. J., González-Gallego, J., and Mauriz, J. L. (2015). Melatonin and endoplasmic reticulum stress: relation to autophagy and apoptosis. J. Pineal Res. 59, 292-307. doi: 10.1111/jpi.12264

Filomeni, G., De Zio, D., and Cecconi, F. (2015). Oxidative stress and autophagy: the clash between damage and metabolic needs. Cell Death Differ. 22, 377-388. doi: $10.1038 /$ cdd.2014.150

Flaskos, J., McLean, W. G., Fowler, M. J., and Hargreaves, A. J. (1998). Tricresyl phosphate inhibits the formation of axon-like processes and disrupts neurofilaments in cultured mouse N2a and rat PC12 cells. Neurosci. Lett. 242, 101-104. doi: 10.1016/s0304-3940(98)00054-8

Foil, L. D., Chambers, H. W., Stinson, R. S., and Glick, B. (1980) Immunological aspects of tri-o-tolyl phosphate-induced delayed neurotoxicity in chickens. Toxicol. Appl. Pharmacol. 56, 259-264. doi: 10.1016/0041-008x(80) 90297-5 
Frake, R. A., Ricketts, T., Menzies, F. M., and Rubinsztein, D. C. (2015). Autophagy and neurodegeneration. J. Clin. Invest. 125, 65-74. doi: 10.1172/JCI73944

Fu, J., Zhao, S. D., Liu, H. J., Yuan, Q. H., Liu, S. M., Zhang, Y. M., et al. (2011). Melatonin promotes proliferation and differentiation of neural stem cells subjected to hypoxia in vitro. J. Pineal Res. 51, 104-112. doi: 10.1111/j. 1600-079x.2011.00867.x

Gage, F. H., and Temple, S. (2013). Neural stem cells: generating and regenerating the brain. Neuron 80, 588-601. doi: 10.1016/j.neuron.2013.10.037

Hardeland, R. (2008). Melatonin, hormone of darkness and more: occurrence, control mechanisms, actions and bioactive metabolites. Cell. Mol. Life Sci. 65, 2001-2018. doi: 10.1007/s00018-008-8001-x

$\mathrm{He}, \mathrm{C}$, and Klionsky, D. J. (2009). Regulation mechanisms and signaling pathways of autophagy. Annu. Rev. Genet. 43, 67-93. doi: 10.1146/annurev-genet102808-114910

Huang, Y. C., Yu, H. S., and Chai, C. Y. (2015). Roles of oxidative stress and the ERK1/2, PTEN and p70S6K signaling pathways in arseniteinduced autophagy. Toxicol. Lett. 239, 172-181. doi: 10.1016/j.toxlet.2015. 09.022

Janjetovic, Z., Nahmias, Z. P., Hanna, S., Jarrett, S. G., Kim, T. K., Reiter, R. J., et al. (2014). Melatonin and its metabolites ameliorate ultraviolet B-induced damage in human epidermal keratinocytes. J. Pineal Res. 57, 90-102. doi: 10.1111/jpi. 12146

Jo, C., Kim, S., Cho, S. J., Choi, K. J., Yun, S. M., Koh, Y. H., et al. (2014). Sulforaphane induces autophagy through ERK activation in neuronal cells. FEBS Lett. 588, 3081-3088. doi: 10.1016/j.febslet.2014. 06.036

Klionsky, D. J., Abeliovich, H., Agostinis, P., Agrawal, D. K., Aliev, G., Askew, D. S., et al. (2008). Guidelines for the use and interpretation of assays for monitoring autophagy in higher eukaryotes. Autophagy 4, 151-175. doi: 10.4161 /auto. 5338

Kovacic, P. (2003). Mechanism of organophosphates (nerve gases and pesticides) and antidotes: electron transfer and oxidative stress. Curr. Med. Chem. 10, 2705-2709. doi: 10.2174/0929867033456314

Lamont, K., Nduhirabandi, F., Adam, T., Thomas, D. P., Opie, L. H., and Lecour, S. (2015). Role of melatonin, melatonin receptors and STAT3 in the cardioprotective effect of chronic and moderate consumption of red wine. Biochem. Biophys. Res. Commun. 465, 719-724. doi: 10.1016/j.bbrc.2015. 08.064

Levine, B., and Kroemer, G. (2008). Autophagy in the pathogenesis of disease. Cell 132, 27-42. doi: 10.1016/j.cell.2007.12.018

Liu, M. L., Wang, J. L., Wei, J., Xu, L. L., Yu, M., Liu, X. M., et al. (2015). Triortho-cresyl phosphate induces autophagy of rat spermatogonial stem cells. Reproduction 149, 163-170. doi: 10.1530/rep-14-0446

Liu, X., Xu, L., Shen, J., Wang, J., Ruan, W., Yu, M., et al. (2016). Involvement of oxidative stress in tri-ortho-cresyl phosphate-induced autophagy of mouse Leydig TM3 cells in vitro. Reprod. Biol. Endocrinol. 14:30. doi: 10.1186/s12958016-0165-x

Long, D. X., Hu, D., Wang, P., and Wu, Y. J. (2014). Induction of autophagy in human neuroblastoma SH-SY5Y cells by tri-ortho-cresyl phosphate. Mol. Cell. Biochem. 396, 33-40. doi: 10.1007/s11010-014-2139-7

Long, D. X., and Wu, Y. J. (2008). Growth inhibition and induction of G(1) phase cell cycle arrest in neuroblastoma SH-SY5Y cell by tri-ortho-cresyl phosphate. Toxicol. Lett. 181, 47-52. doi: 10.1016/j.toxlet.2008.06.871

Lorke, D. E., Stegmeier-Petroianu, A., and Petroianu, G. A. (2017). Biologic activity of cyclic and caged phosphates: a review. J. Appl. Toxicol. 37, 13-22. doi: $10.1002 /$ jat.3369

Parzych, K. R., and Klionsky, D. J. (2014). An overview of autophagy: morphology, mechanism, and regulation. Antioxid. Redox Signal. 20, 460-473. doi: $10.1089 /$ ars. 2013.5371

Pi, H., Xu, S., Reiter, R. J., Guo, P., Zhang, L., Li, Y., et al. (2015). SIRT3SOD2-mROS-dependent autophagy in cadmium-induced hepatotoxicity and salvage by melatonin. Autophagy 11, 1037-1051. doi: 10.1080/15548627.2015. 1052208
Reiter, R. J. (1991). Pineal melatonin: cell biology of its synthesis and of its physiological interactions. Endocr. Rev. 12, 151-180. doi: 10.1210/edrv12-2-151

Song, J., Kang, S. M., Lee, K. M., and Lee, J. E. (2015). The protective effect of melatonin on neural stem cell against LPS-induced inflammation. Biomed Res. Int. 2015:854359. doi: 10.1155/2015/854359

Song, F., Kou, R., Zou, C., Gao, Y., Zeng, T., and Xie, K. (2014). Involvement of autophagy in tri-ortho-cresyl phosphate- induced delayed neuropathy in hens. Neurochem. Int. 64, 1-8. doi: 10.1016/j.neuint.2013.10.017

Sou, Y. S., Tanida, I., Komatsu, M., Ueno, T., and Kominami, E. (2006). Phosphatidylserine in addition to phosphatidylethanolamine is an in vitro target of the mammalian Atg8 modifiers, LC3, GABARAP and GATE-16. J. Biol. Chem. 281, 3017-3024. doi: 10.1074/jbc.m505888200

Torres, F., Gonzalez-Candia, A., Montt, C., Ebensperger, G., Chubretovic, M. Seron-Ferre, M., et al. (2015). Melatonin reduces oxidative stress and improves vascular function in pulmonary hypertensive newborn sheep. J. Pineal Res. 58, 362-373. doi: 10.1111/jpi.12222

Tsukamoto, A., Uchida, N., Capela, A., Gorba, T., and Huhn, S. (2013). Clinical translation of human neural stem cells. Stem Cell Res. Ther. 4:102 doi: $10.1186 /$ scrt313

Wang, P., Sun, X., Wang, N., Tan, D. X., and Ma, F. (2015). Melatonin enhances the occurrence of autophagy induced by oxidative stress in Arabidopsis seedlings. J. Pineal Res. 58, 479-489. doi: 10.1111/jpi.12233

Wang, F. W., Wang, Z., Zhang, Y. M., Du, Z. X., Zhang, X. L., Liu, Q., et al. (2013). Protective effect of melatonin on bone marrow mesenchymal stem cells against hydrogen peroxide-induced apoptosis in vitro. J. Cell. Biochem. 114, 2346-2355. doi: $10.1002 / j c b .24582$

Wolkoff, P., Crump, D. R., and Harrison, P. T. (2016). Pollutant exposures and health symptoms in aircrew and office workers: is there a link? Environ. Int. 87, 74-84. doi: 10.1016/j.envint.2015.11.008

Xu, L. L., Liu, M. L., Wang, J. L., Yu, M., and Chen, J. X. (2016). Saligenin cyclic-otolyl phosphate (SCOTP) induces autophagy of rat spermatogonial stem cells. Reprod. Toxicol. 60, 62-68. doi: 10.1016/j.reprotox.2016.01.004

Xu, H. Y., Wang, P., Sun, Y. J., Jiang, L., Xu, M. Y., and Wu, Y. J. (2017). Autophagy in Tri-o-cresyl phosphate-induced delayed neurotoxicity. J. Neuropathol. Exp. Neurol. 76, 52-60. doi: 10.1093/jnen/nlw108

Yoo, Y. M., Han, T. Y., and Kim, H. S. (2016). Melatonin suppresses autophagy induced by clinostat in preosteoblast MC3T3-E1 cells. Int. J. Mol. Sci. 17:526. doi: 10.3390/ijms17040526

Yorimitsu, T., and Klionsky, D. J. (2005). Autophagy: molecular machinery for self-eating. Cell Death Differ. 12, 1542-1552. doi: 10.1038/sj.cdd.4401765

Yu, L., Sun, Y., Cheng, L., Jin, Z., Yang, Y., Zhai, M., et al. (2014). Melatonin receptor-mediated protection against myocardial ischemia/reperfusion injury: role of SIRT1. J. Pineal Res. 57, 228-238. doi: 10.1111/jpi.12161

Zhang, W. L., Meng, H. Z., Yang, R. F., Yang, M. W., Sun, G. H., Liu, J. H., et al. (2016). Melatonin suppresses autophagy in type 2 diabetic osteoporosis. Oncotarget 7, 52179-52194. doi: 10.18632/oncotarget.10538

Zhang, L. P., Wang, Q. S., Guo, X., Zhu, Y. J., Zhou, G. Z., and Xie, K. Q. (2007). Time-dependent changes of lipid peroxidation and antioxidative status in nerve tissues of hens treated with tri-ortho-cresyl phosphate (TOCP). Toxicology 239, 45-52. doi: 10.1016/j.tox.2007.06.091

Conflict of Interest: The authors declare that the research was conducted in the absence of any commercial or financial relationships that could be construed as a potential conflict of interest.

Copyright $\odot 2020 \mathrm{Liu}, \mathrm{Zhou}, \mathrm{Li}$, Ren, Li, Li, Liu, Song, Hao and Wang. This is an open-access article distributed under the terms of the Creative Commons Attribution License (CC BY). The use, distribution or reproduction in other forums is permitted, provided the original author(s) and the copyright owner(s) are credited and that the original publication in this journal is cited, in accordance with accepted academic practice. No use, distribution or reproduction is permitted which does not comply with these terms. 